\title{
Rastreabilidade e Análise de Impacto: Um caso de aplicação utilizando a ferramenta Visual Studio Team System
}

\author{
Tiago Domenici Griffo ${ }^{1}$, Gothardo Francisco de Magalhães Santos ${ }^{1}$, Rodrigo Becke \\ Cabral $^{1}$ \\ ${ }^{1}$ Cadsoft Informática Ltda \\ Belo Horizonte - MG - Brasil \\ \{tiago.griffo, gothardo, rodrigo.cabral\}@cadsoft.com.br
}

\begin{abstract}
This paper describes the deployment of Microsoft Visual Studio Team System (VSTS) in the software development process at Cadsoft supporting the requirements management discipline on the execution of impact analysis and traceability of products artifacts, reaching an expected result of MPS.BR level G.
\end{abstract}

Resumo. Este artigo apresenta a implantação da ferramenta Microsoft Visual Studio Team System (VSTS) no processo de desenvolvimento de software da Cadsoft, apoiando a disciplina gerência de requisitos na realização da análise de impacto e rastreabilidade de artefatos de produtos, atendendo a um resultado esperado do nível $G$ do MPS.BR.

\section{Introdução}

A Cadsoft é uma empresa que atua há vinte anos no setor educacional. Eventos recentes no contexto desse setor evidenciam mudanças fundamentais, que estão chegando com intensidade ao negócio das instituições de ensino superior brasileiras. Estudos conduzidos pelo MEC obtiveram conclusões surpreendentes centradas no seguinte fato: as instituições de ensino estão entrando em uma nova fase evolutiva, induzidas pelo ambiente em que estão inseridas (SILVA e CABRAL, 2008). Essa nova fase exigiria uma postura diferente do gestor, executivo e técnico da educação, voltada para uma administração profissional de suas instituições de ensino superior.

Diante desse cenário, a Cadsoft procurou melhorar o seu processo de desenvolvimento de software para atender com mais qualidade e agilidade o setor educacional, sempre se preocupando em gerar um produto confiável e seguro que agregue valor ao negócio dos seus clientes.

Em outubro de 2007, a Cadsoft ingressou no grupo G3 do programa MPS.BR (melhoria de processo do software brasileiro) firmando uma parceria com a Fumsoft (Sociedade Mineira de Software) e a SOFTEX (Associação para Promoção da Excelência do Software Brasileiro), com o objetivo de implantar os processos de Gerência de Projetos e Gerência de Requisitos e a obtenção da certificação no nível G desse programa em seu serviço de Evolução de Produto.

A partir daí, foram realizadas uma série de reuniões de consultoria e projetos internos com o objetivo de definir o processo produtivo da Cadsoft e escolher as ferramentas que o apoiariam. 
Um problema crítico observado em nosso processo produtivo foi a criação de novas funcionalidades ou a alteração de uma já existente, gerando impactos positivos e negativos em nossos produtos. Nem sempre esses impactos são totalmente analisados ou verificados, gerando novas falhas que poderiam ser facilmente detectadas antes de liberada a versão para o cliente.

Para resolver esse problema e atender um resultado esperado do processo de Gerência de Requisitos do nível $G$ do MPS.BR, foi realizada a implantação da ferramenta Microsoft Visual Studio Team System (VSTS, 2009) que possibilita um controle fácil e ágil das solicitações de mudança no produto e dos seus requisitos, proporcionando rastreabilidade horizontal e vertical dos requisitos e análise de impacto das mudanças realizadas.

\section{Serviço Evolução de Produto}

\subsection{MPS.BR}

Segundo o Guia Geral, versão 1.2, o MPS.BR é um programa para Melhoria de Processo do Software Brasileiro coordenado pela Associação para Promoção da Excelência do Software Brasileiro (SOFTEX).

A base técnica para construção e evolução do modelo é composta pelas normas NBR ISO/IEC 12207 - Processo de Ciclo de Vida de Software, ISO/IEC 15504 - Avaliação de Processo e pelo CMMI-Dev. Toda avaliação MPS.BR verifica se uma organização ou parte dela se encontra em conformidade com os processos do MR-MPS (modelo de referência) e é realizada utilizando o processo e o método de avaliação descritos no MAMPS.

O nível $G$ do MPS.BR é o primeiro nível de maturidade do MR-MPS. Sua implementação deve ser executada com cautela por estabelecer o início dos trabalhos em implantação de melhoria dos processos de software na organização. Ao final da implantação desse nível a organização deve ser capaz de gerenciar parcialmente seus projetos de desenvolvimento de software.

Dois pontos são desafiadores na implantação do nível G: (1) mudança de cultura organizacional, orientando a definição e melhoria dos processos de desenvolvimento de software; (2) definição do conceito acerca do que é "projeto" para a organização.

\subsection{Modelo Serviço Tarefa}

O modelo Serviço Tarefa foi criado pela Cadsoft com o objetivo de definir, documentar e padronizar os serviços prestados ao seu foco.

O modelo baseia-se nos seguintes pressupostos:

- Serviços simples e tarefas complexas;

- Serviço começa na necessidade do cliente e termina no atendimento dessa necessidade, sendo norteado por métricas;

- Um serviço sempre se desenvolve por meio de projetos;

- Tarefa é um elemento unitário na orquestração do serviço;

- O objetivo da tarefa é produzir um resultado que contribua para a entrega do serviço; 
- A tarefa possui um CHA (conhecimentos, habilidades e atitudes) necessário para que seja executada;

- Toda tarefa possui entradas e saídas bem definidas;

- Projeto é a instância de um serviço que possui início, meio e fím e tem um resultado único.

\subsection{Serviço Evolução de Produto}

O serviço Evolução de Produto (EPROD) prestado pela Cadsoft é responsável por criar ou incorporar mudanças às suas soluções tecnológicas - seus produtos. Seu objetivo é transformar em realidade a necessidade de adequação do produto ao negócio, de forma planejada e com qualidade. Essa transformação é feita por meio de projetos bem definidos, sempre em contato com o cliente, avaliando sua satisfação em cada entrega.

O serviço EPROD possui um ciclo de vida com quatro fases: Concepção, Elaboração, Construção e Transição. No final de cada fase é executada a tarefa Revisar Marcos para determinar se os objetivos da fase foram alcançados. As fases indicam também a ênfase dada à execução de uma tarefa em um dado instante do projeto, conforme pode ser observado na tabela 1.

Tabela 1. Tarefas do serviço Evolução de Produto x Esforço na execução de cada tarefa

\begin{tabular}{|c|c|c|c|c|}
\hline \multirow{2}{*}{ Tarefas } & \multicolumn{4}{|c|}{ Fases } \\
\hline & Concepcão & Elaboracão & Construcão & Transicão \\
\hline Identificar Necessidade & MIVIDDAX & $(1 / 7 / 270$ & $19177 \mathrm{Th}$ & \\
\hline Formalizar Necessidade & $\mathbb{H} / \mathbb{W} / \mathrm{D}$ & $1017 \mathrm{~T}$ & (1/17/7m & \\
\hline Analisar e Propor Solução & $D \mathbb{D}$ & 1171771 & $117 / 7 \mathrm{M}$ & \\
\hline Especificar Requisitos & $\square \mathbb{D O D}$ & 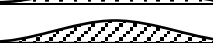 & 001000 & \\
\hline Montar Plano de Projeto & $\mathscr{D} \mathbb{V} / \mathbb{D}$ & 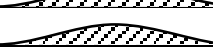 & nomens & \\
\hline Realizar Abertura do Projeto & $\mathbb{Z 1 / N A}$ & & & \\
\hline Criar Casos de Teste & 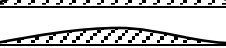 & 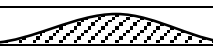 & 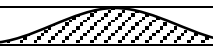 & $117 / 7 / 2$ \\
\hline Desenvolver Solução & $\infty x$ & 501000 & 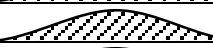 & DIVID \\
\hline Verificar Solução & $0017 / 20$ & - & $\triangle 1 / D D$ & \\
\hline Criar Documentação Funcional & 00000000 & 0000000 & $\mathbb{W I O D}$ & $W 101000$ \\
\hline Integrar Mudanças & +10101010 & $10117 \mathrm{~mm}$ & $M \mathbb{N} / 1$ & DIVIDD \\
\hline Liberar Produto/Versão & nollom & (17/7hn & DIVID & \\
\hline Homologar Solução & & 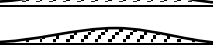 & $\triangle D / D D$ & \\
\hline Encerrar Projeto & & & & \\
\hline Realizar Entregas do Projeto & $\infty 117170$ & & $\infty$ & \\
\hline Revisar Marcos & & IIIV & & \\
\hline
\end{tabular}

As tarefas do serviço EPROD que trabalham diretamente os requisitos, objeto de estudo desse artigo, são: Especificar Requisitos, Desenvolver Solução, Verificar Solução, Integrar Mudanças, Liberar Produto/Versão e Homologar Solução.

\section{VSTS - Visual Studio Team System}

O VSTS possui um sistema de rastreabilidade de trabalho de equipes de desenvolvimento. O sistema é composto por work items (itens de trabalho) que representam uma unidade de trabalho. Esses, por sua vez, possuem um fluxo de trabalho associado e uma árvore de funcionalidades que agrupa os itens de trabalho logicamente.

O fluxo de trabalho indica quais são as possíveis mudanças de estados de um item de trabalho, bem como as restrições aplicadas a essas mudanças. 
Todo item de trabalho é associado a um item na árvore de funcionalidades que representa hierarquicamente a estrutura dos produtos.

Pode-se dividir a base de informações do VSTS em dois componentes: uma base de dados operacional e um armazém de dados. A base operacional é o local onde são armazenados os dados da operação imediata do sistema, ou seja, a base de informações "viva" do sistema. O armazém de dados contém uma visão congelada e não normalizada dos dados provenientes da base operacional e é atualizada automaticamente.

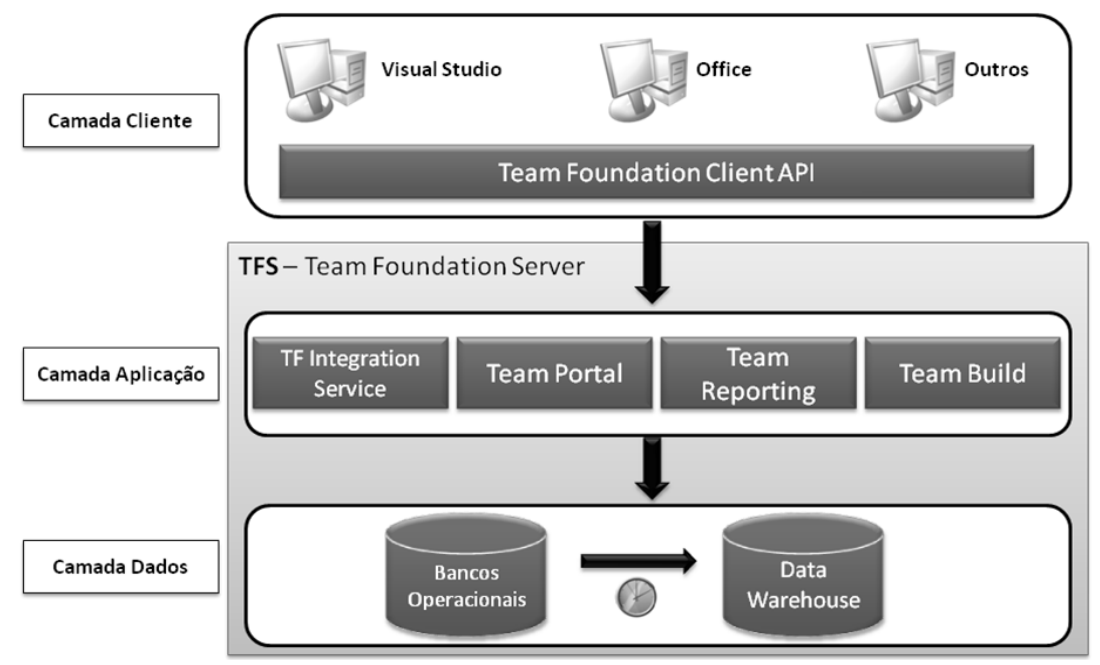

Figura 1. Um visão da arquitetura do VSTS

Os tipos de itens de trabalho definidos para o serviço Evolução de Produto são: Solicitação de Mudança, Requisito e Falha.

\subsection{Solicitação de Mudança}

Esse tipo de item de trabalho registra as solicitações de mudança nos produtos da Cadsoft. Toda mudança nos produtos é justificada através do registro de solicitação de mudança vinculado a ela. Esse tipo de item de trabalho é associado a árvore de funcionalidades e contém a informação de qual serviço irá executar a mudança no produto.

\subsection{Requisito}

Esse tipo de item de trabalho registra os requisitos (funcionais e não funcionais) de software dos produtos. Esse tipo de item de trabalho é associado a árvore de funcionalidades e permite a rastreabilidade horizontal (entre requisitos) e vertical (requisito $\mathrm{x}$ funcionalidade e requisito $\mathrm{x}$ código fonte). Por meio dessa amarração é possível realizar a análise de impacto de uma mudança solicitada no produto pelo cliente.

\subsection{Falha}

Esse tipo de item de trabalho registra as falhas de software encontradas durante a execução das tarefas Verificar Solução e Homologar Solução. Esse tipo de item de trabalho é associado a árvore de funcionalidades. Com essa informação é possível definir métricas de qualidade e aceitação do produto. 


\subsection{Visões}

Visões ou queries são formas de consultar a base de dados operacional de itens de trabalho. Existem consultas pré-definidas e as que podem ser customizadas pela equipe, de acordo com a necessidade da empresa. Nesse tipo de consulta não é possível fazer associação entre diferentes tipos de itens de trabalho. É possível criar consultas filtrando os itens de trabalho pelo conteúdo dos seus campos ou pelo seu estado.

\subsection{Relatórios}

Relatórios são formas de consultar o armazém de dados do TFS (Team Foundation Server). O armazém de dados possui cubos pré-definidos que permite a equipe customizar relatórios para atender as necessidades de informação da empresa ou dos clientes.

\section{Aplicação do VSTS nas tarefas do Serviço Evolução de Produto}

Durante a execução de um projeto do serviço EPROD, a primeira tarefa envolvendo requisitos é chamada Especificar Requisitos. Essa tarefa tem por objetivo traduzir a necessidade do cliente e a solução proposta em requisitos funcionais e não funcionais que definirão o desenvolvimento a ser executado, o qual necessariamente deverá agregar valor e evoluir o negócio do cliente.

Ao iniciar uma implementação de um projeto ou iteração, ou seja, na execução da tarefa Desenvolver Solução, o desenvolvedor tem como itens de trabalho requisitos. Nesse momento cada requisito tem seu estado modificado, indicando o ponto onde se encontra no processo: Em desenvolvimento. Ao finalizar o desenvolvimento, o desenvolvedor realiza os testes necessários para verificar se a implementação atendeu todos os requisitos, executando a tarefa Verificar Solução. O estado do item de trabalho é alterado para Em teste.

Caso seja encontrada alguma não conformidade entre a implementação e os requisitos, é criado um item de trabalho do tipo Falha que deverá ser resolvido antes da integração do código à base oficial de produtos.

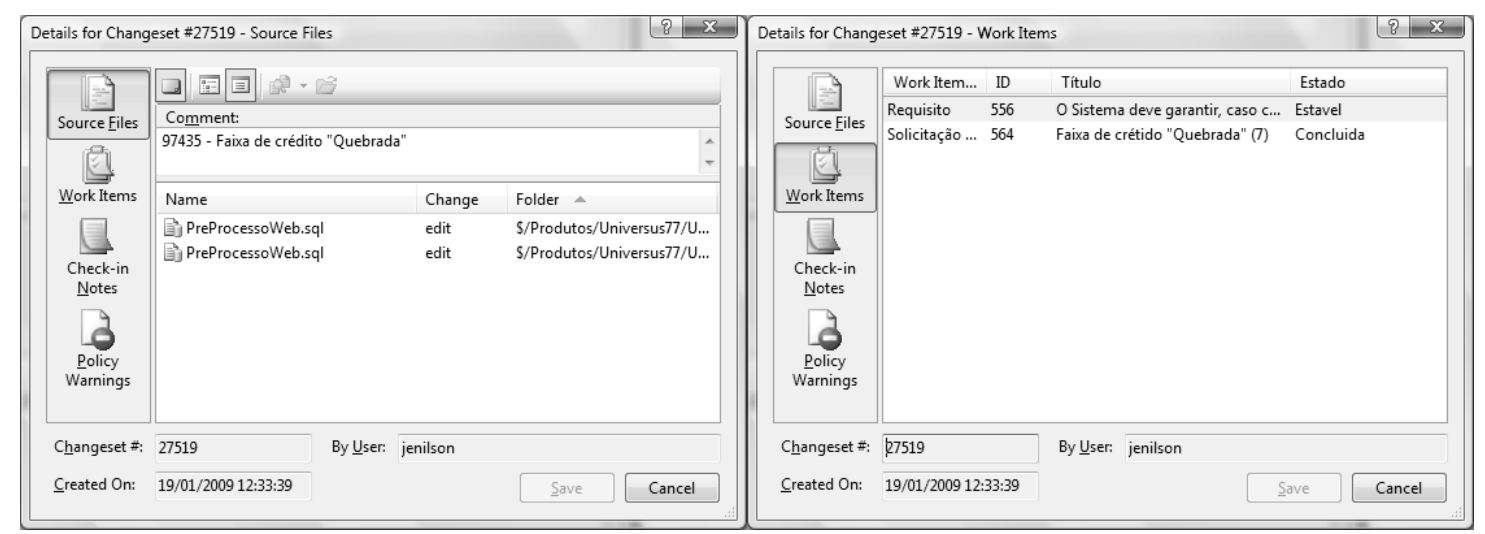

Figura 2. Detalhes de uma operação de check-in com requisito e solicitação de mudança associados

Finalizada a tarefa Verificar Solução, o desenvolvedor integra o código modificado à base oficial de produtos no VSTS. Nesse momento os requisitos e solicitações de 
mudança são associados ao código fonte. Esse vínculo possibilita a realização da análise de impacto em mudanças futuras nesses artefatos, conforme figura 2.

O produto binário é gerado e disponibilizado ao cliente. Os requisitos têm seu estado alterado para Em homologação. O cliente realiza a homologação da solução e os itens de trabalho têm seu estado alterado para Estável, finalizando o ciclo de vida dos requisitos em um projeto.

Durante a execução do serviço EPROD, existem dois grandes momentos nos quais a análise do impacto deve ser feita. Primeiro, quando se especifica os requisitos para um projeto EPROD, novos requisitos serão criados e requisitos existentes serão modificados dentro de um produto. Nesse momento fica clara a necessidade de análise do impacto causada pela modificação que será inserida no produto. Uma modificação inicialmente considerada pequena pode ter sua percepção de proporção alterada após a realização da análise de impacto. O segundo momento é quando, durante o andamento do projeto, ocorre uma mudança de escopo e essa deve ter seu impacto analisado, para que os ajustes no projeto sejam feitos em relação ao custo, prazo, recursos, dentre outros.

A análise de impacto consiste na geração de relatórios do VSTS que consultam o armazém de dados relacionando os requisitos, falhas, solicitações de mudança com os códigos fonte e as funcionalidades. Como resultado, é gerado um relatório de possíveis impactos de uma mudança. A análise de impacto pode ter como ponto de partida um código fonte, uma funcionalidade ou um item de trabalho. Após a geração dos relatórios é necessária uma interpretação por parte dos envolvidos na mudança, para que a análise de impacto esteja completa e o tamanho e impacto da mudança sejam conhecidos.

\section{Informações sobre o processo de implantação do VSTS}

O processo de implantação do VSTS ocorreu no formato de um projeto, onde foram consideradas tarefas de concepção, apresentação e aprimoramento do processo. Após essa primeira fase foi realizada a migração dos códigos-fonte, a composição de guias e manuais de operação e treinamentos à equipe, conforme apresentado na tabela 2 .

Tabela 2. Cronograma de implantação do VSTS

\begin{tabular}{|l|l|l|}
\hline Tarefa & Datas & Recursos Envolvidos \\
\hline Reunião de concepção do projeto & $13 / 08 / 2008$ & Equipe do projeto \\
\hline Planejamento & 13 a $20 / 08 / 2008$ & Equipe do projeto \\
\hline Montagem do Processo - Piloto & $21 / 08$ a 25/09/2008 & Equipe do projeto \\
\hline Apresentação do Piloto & $26 / 09 / 2008$ & Equipe do projeto e gestores da Cadsoft \\
\hline Adequações no Processo & $26 / 09$ a $07 / 10 / 2008$ & Equipe do projeto \\
\hline Criação de Guias e Manuais & 08 a $15 / 10 / 2008$ & Equipe do projeto \\
\hline Migração dos Códigos-fonte e Go-live & $16 / 10 / 2008$ & Equipe do projeto \\
\hline Suporte a operação e Mentoring & 17 a $24 / 10 / 2008$ & Equipe do projeto e colaboradores da fábrica \\
\hline Treinamentos & 03 a 05/11/2008 & Equipe do projeto e colaboradores da fábrica \\
\hline
\end{tabular}

A equipe do projeto foi formada por dois colaboradores, membros do Grupo de Engenharia de Processo de Software (Software Engineering Process Group - SEPG) da Cadsoft, com conhecimento no processo produtivo da empresa e na ferramenta VSTS.

O investimento realizado no projeto foi de $\mathrm{R} \$ 23.248,87$, conforme tabela 3. 
Tabela 3. Investimento do projeto

\begin{tabular}{|l|r|}
\hline Descrição & Investimento \\
\hline Esforço da equipe do projeto & $\mathrm{R} \$ 17.336,20$ \\
\hline Hardware & $\mathrm{R} \$ 2.416,67$ \\
\hline Treinamento da equipe na ferramenta & $\mathrm{R} \$ 3.496,00$ \\
\hline
\end{tabular}

Não houve necessidade de investimento na ferramenta VSTS, já que a Cadsoft possui o nível de parceria Gold Certified Partner e um dos benefícios é o licenciamento gratuito da ferramenta em questão.

\section{Conclusão}

Implantar um processo de software é uma tarefa muito maior quando comparada à instalação de uma ferramenta, por mais complexa que ela seja. A definição do processo em si, as reuniões para se chegar a um consenso sobre a melhor maneira de se realizar uma tarefa, a documentação dos resultados, a aderência dos resultados ao modelo sendo seguido e o aculturamento da equipe ao processo desenvolvido são tarefas que demandam tempo e devem ser feitas por pessoas competentes e dispostas a mudar suas opiniões.

Contudo, muitas vezes a falta de uma ferramenta adequada pode inviabilizar a execução de um processo definido. Existem ferramentas flexíveis que aderem aos mais diversos modelos. É importante, porém, planejar a implantação das ferramentas de forma metódica sempre se atendo ao atendimento dos requisitos do modelo e a adaptação da equipe à utilização da ferramenta.

Com o ingresso da Cadsoft no programa MPS.BR, iniciou-se a profissionalização do seu processo de desenvolvimento de software. O uso de uma ferramenta para documentação dos requisitos de produto permitiu que o conhecimento, antes somente presente nos colaboradores, fosse formalizado dentro do VSTS.

O VSTS entrou em produção em Outubro de 2008 e até Março de 2009 já existem mais de 500 requisitos registrados, conforme mostrado na figura 4.

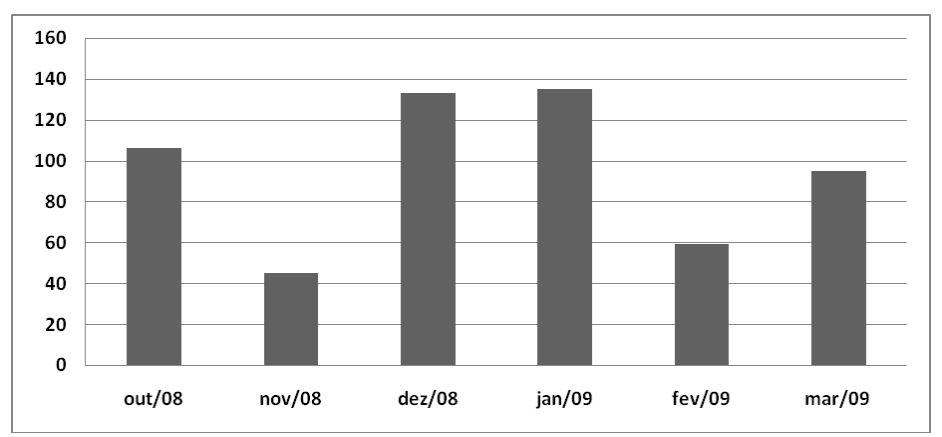

Figura 3. Gráfico de registro de requisitos por mês

Com isso, o impacto das mudanças que antes era somente analisado por meio do conhecimento especializado, muitas das vezes desmembrado em diversos colaboradores, está agora disponível para todos os colaboradores na ferramenta VSTS, sendo possível minimizar o viés subjetivo da análise. 
Pode-se observar no gráfico da figura 5 que os pedidos de suporte à operação da ferramenta foram gradativamente diminuindo à medida que a equipe se adaptou ao VSTS, mostrando claramente a curva de aprendizado dos colaboradores.

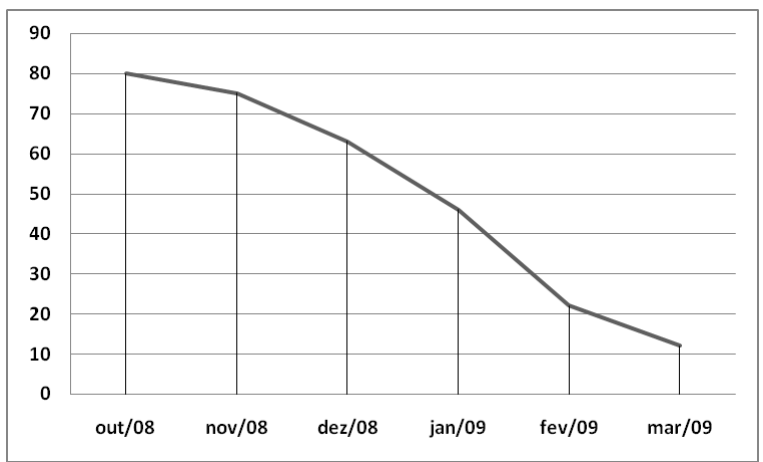

Figura 4. Gráfico de registro de suporte à operação do VSTS por mês

A melhoria no processo de rastreabilidade e análise de impacto ainda está no início, porém os ganhos já são perceptíveis tanto pela Cadsoft quanto pelos seus clientes. As mudanças estão sendo entregues com uma melhor qualidade, provenientes de um processo claramente definido e de conhecimento de todos. Um dos principais resultados tangíveis desse trabalho foi o desenvolvimento de um documento de novidades da versão, em um formato integrado ao processo produtivo e com facilidade de geração instantânea de relatórios.

\section{Referências Bibliográficas}

ASSOCIAÇÃO BRASILEIRA DE NORMAS TÉCNICAS. NBR 6023: Informação e Documentação - Referências - Elaboração. Rio de Janeiro: ABNT, 2000.

CABRAL, Rodrigo Becke et al. Serviço Evolução de Produto. Belo Horizonte, 2008. 25 p.

GRIFFO, Tiago Domenici. Ferramentas de Apoio ao Processo de Software. 2008. $51 \mathrm{f}$. Monografia (Graduação) - Curso de Ciência da Computação, Departamento de Ciências Exatas e Tecnologia, Centro Universitário de Belo Horizonte, Belo Horizonte, 2008.

SILVA, Daniel Fernando Antonucci e; CABRAL, Rodrigo Becke. Modelo de Maturidade de Processos de Gestão Acadêmica para Instituições Privadas de Ensino Superior. In: ENANGRAD, 19, 2008, Curitiba. Anais... . São Paulo, 2008. p. 1 - 18.

SOFTEX. MPS.BR - Melhoria de Processo do Software Brasileiro: Guia Geral (Versão 1.2). Disponível em: <http://www.softex.br/mpsBr/_guias/default.asp $>$. Acesso em: 15 mar. 2008.

VSTS. Microsoft Visual Studio Team System - Home Page. Disponível em: $<$ http://msdn.microsoft.com/pt-br/teamsystem/default.aspx $>$. Acesso em: 27 mar. 2009. 